\title{
Invaginación intestinal recurrente secundaria a apéndice cecal invaginado. Caso clínico
}

\author{
CLAUDIA NORAMBUENA C. ${ }^{1}$, ANDREA RUIZ C. ${ }^{1}$, JAQUELINE YÁÑEZ V. ${ }^{2}$ \\ 1. Médico. Residente Cirugía Pediátrica Universidad de Chile. Hospital Exequiel González Cortés. \\ 2. Médico. Cirujano Pediátrico. Hospital Exequiel González Cortés.
}

\begin{abstract}
Recurrent intussusception secondary to invaginated cecal appendix. Case report

Introduction: Intussusception is an idiopathic pathology that accounts for $95 \%$ of cases found; the remaining $5 \%$ is caused by some triggering events. Recurrences occur in 7 to $12 \%$ of cases and are associated with multiple causes. The aim of this research is to present the case of a patient with recurrent intussusception and analyze the cause of the recurrence. Case report: The patient was a 16 month-old male infant with a history of first episode of intussusception surgery at 4 months old. His second intussusception episode took place at 9 months of age, which was treated with pneumatic reduction process; at 16 months of age he presented his third episode, which was treated with hydrostatic reduction process. 8 hours post procedure, the patient had a new intussusception condition and surgical manual reduction was performed. Ileocolic intussusception was found with an appendiceal intrathecal remnant acting as the invaginating head. Remnant was removed and sent for biopsy. Conclusions: Recurrent intussusception is associated with triggering anatomical factors; therefore, in the case of recurrences, a possible organic cause should be considered and surgical treatment is recommended. (Key words: Recurrent intussusception, appendiceal remnant, pneumatic reduction, hydrostatic reduction). Rev Chil Pediatr 2012; 83 (6): 595-598
\end{abstract}

\section{RESUMEN}

Introducción: La invaginación intestinal es una patología de causa idiopática en el 95\% de los casos, encontrándose en el 5\% restante algún factor desencadenante que la justifique. Las recurrencias se presentan en un 7 a $12 \%$ de los casos y se asocian a múltiples causas. El objetivo de este trabajo es presentar el caso de un paciente con invaginación intestinal recurrente y analizar la causa de la recurrencia. Caso clínico: Paciente de 1 año y 4 meses de edad, sexo masculino, con antecedente de primer episodio de Invaginación intestinal operado a los 4 meses de edad. Presenta segundo episodio de invaginación a los 9 meses de edad, la cual se trató con desinvaginación neumática y a los 16 meses de edad presentó su tercer episodio, manejandose con desinvaginación hidrostática. Ocho horas post procedimiento el paciente presentó nueva invaginación; se realizó reducción manual quirúrgica. Se encontró una invaginación ileocólica con un remanente apendicular intracecal

Recibido el 16 de enero de 2012, devuelto para corregir el 09 de marzo de 2012, segunda versión el 20 de julio de 2012 , aceptado para publicación el 28 de agosto de 2012

Este trabajo cumple con los requisitos sobre consentimiento /asentimiento informado, comité de ética, financiamiento, estudios animales y sobre la ausencia de conflictos de intereses según corresponda.

Correspondencia a:

Claudia Norambuena Cuadra

E-mail: norambuenaclaudia@yahoo.com 
actuando como cabeza invaginante. Se reseco remanente y se envió a biopsia. Conclusiones: La invaginación intestinal recurrente se asocia a factores anatómicos desencadenantes, por lo cual al presentar recurrencias se debe tener en consideración una posible causa orgánica, ante lo cual se recomienda el tratamiento quirúrgico. (Palabras clave: Invaginación recurrente, remanente apendicular, reducción neumática, reducción hidrostática).

Rev Chil Pediatr 2012; 83 (6): 595-598

\section{Introducción}

La invaginación intestinal es una patología de causa idiopática en el $95 \%$ de los casos, encontrándose en el $5 \%$ restante algún factor desencadenante que la origina. Las recurrencias se presentan en un 7 a $12 \%$ de los casos y se asocian a múltiples causas, entre ellas divertículo de Meckel, pólipos intestinales y remanentes apendiculares ${ }^{1}$.

La realización de una apendicectomía incidental ha sido debatida por años. Esta se realiza en casos en que una futura apendicitis sería de alto riesgo o difícil de diagnosticar ${ }^{2}$. Desafortunadamente, al realizar la apendicectomía se incrementan los riesgos de contaminación ${ }^{3}$. Con el fin de prevenir este riesgo, muchos cirujanos realizan una apendicectomía por inversión, técnica que fue descrita por primera vez en 1895 por Edelbohls ${ }^{4}$. Sin embargo, ésta técnica no está exenta de complicaciones y debemos tenerlas en consideración. El objetivo de este trabajo es presentar el caso de un paciente con antecedentes de invaginación intestinal recurrente secundaria a un remanente apendicular, analizar su evolución y tratamientos recibidos.

\section{Caso clínico}

Paciente de 1 año y 4 meses de edad, sexo masculino, quien presentó su primer episodio de Invaginación intestinal, de etiología idiopática, a los 4 meses de edad. Se optó por realizar tratamiento quirúrgico, desinvaginando manualmente y además se decidió realizar una apendicectomía invaginante. Luego de cinco meses, el paciente consultó por cuadro de 36 horas de evolución caracterizado por dolor abdominal tipo cólico, asociado a vómitos y deposiciones diarreicas con estrías san- guinolentas. Se realizó una ecotomografía la cual confirmó el diagnóstico de una extensa invaginación intestinal ileocólica que llegaba hasta el colon descendente (figura 1). Siendo el segundo episodio de invaginación del paciente se decidió tratamiento con desinvaginación neumática (figura 2), la cual fue exitosa siendo el paciente dado de alta al segundo día. A los 16 meses de edad consultó nuevamente por cuadro de dolor abdominal tipo cólico de $24 \mathrm{~h}$ de evolución asociado a deposiciones sanguinolentas. Se realizó una ecotomografía, la cual demostró nuevamente una invaginación intestinal ileocólica, correspondiendo a su tercer episodio (figura 3). En esta oportunidad se decidió manejar con desinvaginación hidrostática (figura 4). Ocho horas posterior al procedimiento, el paciente comenzó nuevamente con dolor abdominal cólico por lo cual se realizó una nueva ecografía en la cual se observó nuevamente una invaginación ileocólica. Siendo el cuarto episodio de invaginación intestinal, se decidió realizar tratamiento quirúrgico con reducción manual de la invaginación. Se realizó una laparotomía supraumbilical transversa derecha encontrándose una invaginación ileocólica, la cual se redujo manualmente. No se encontró apéndice cecal y se palpó en zona de confluencia de las tenias, pero por dentro del lumen del ciego, un cuerpo extraño, por lo cual se realizó una cecotomía. Al abrir ciego se observó una estructura tubular de 2,5 cm de largo sugerente de remanente apendicular, el cual estaba actuando como cabeza invaginante. Se resecó el remanente y se envió a biopsia. Fue dado de alta al $4^{\circ}$ día postoperatorio sin complicaciones. La biopsia describió la presencia de un remanente apendicular. Hasta la fecha el paciente ha presentado una buena evolución, sin nuevos episodios de invaginación intestinal. 


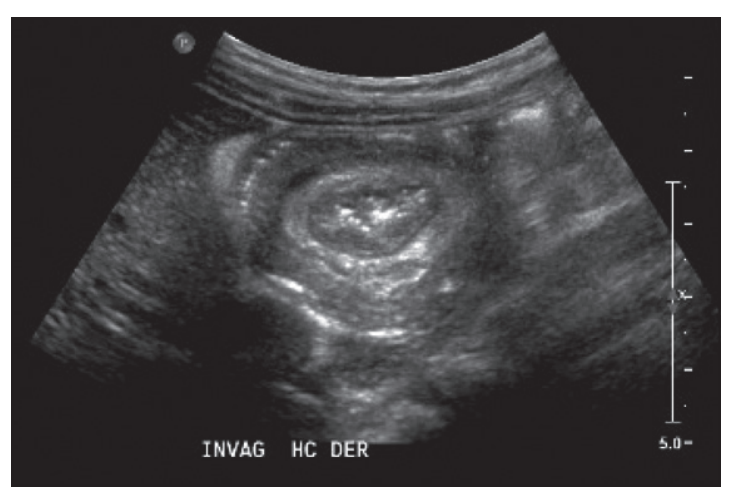

Figura 1. Imágen en "dona”. Borde externo hipoecogénico con centro hiperecogénico.

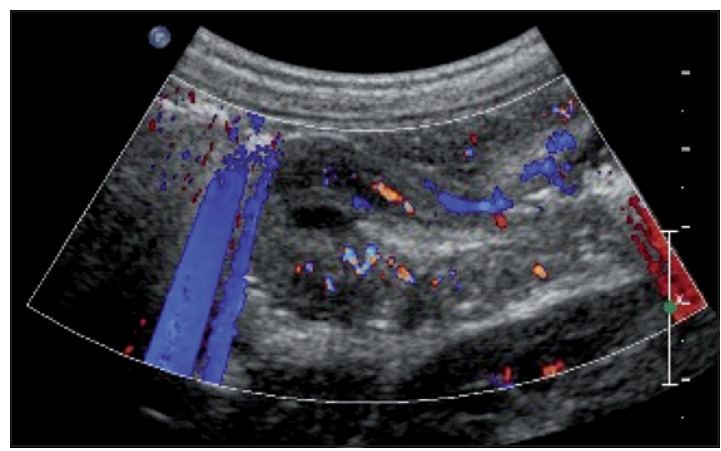

Figura 3. Invaginación ileocólica. Corte longitudinal.

\section{Discusión}

Resulta un desafío diagnóstico el diferenciar si se trata de una invaginación intestinal idiopática o secundaria y su importancia radica en que el enfrentamiento terapéutico va a ser diferente en uno u otro caso. Existen características clínicas y de imagenología que nos permiten un acercamiento al diagnóstico ${ }^{5}$. La edad es uno de los factores a considerar ya que las invaginaciones idiopáticas generalmente se producen entre los 3 y los 12 meses a diferencia de las secundarias que se ven usualmente en niños mayores. Blakelock mostró que el porcentaje de invaginaciones secundarias aumentó de un 5\% en el grupo etario menor de un año a un $60 \%$ entre los 5 y 14 años $^{6}$. La presencia de recurrencias, especialmente más de

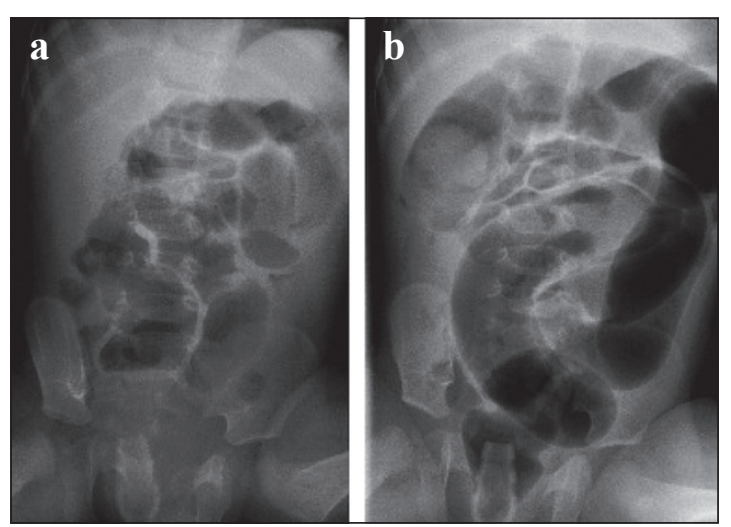

Figura 2. Desinvaginación neumática. a) previo a procedimiento. b) durante procedimiento.

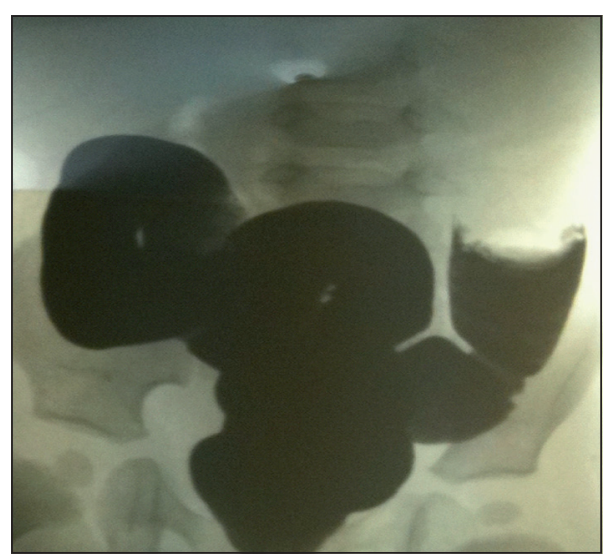

Figura 4. Desinvaginación hidrostática.

una, también es un dato relevante que nos puede orientar hacia la presencia de un elemento patológico que este causando el o los episodios de invaginación ${ }^{7}$. El caso que presentamos justamente presentaba una edad mayor a un año y había presentado más de un episodio de invaginación intestinal. En cuanto al aporte de los exámenes imagenológicos, la ecotomografía es el examen de elección para la detección de alteraciones anatómicas responsables de la invaginación, ya que es capaz de diagnosticarlas entre el $66-75 \%$ de los $\operatorname{casos}^{5,7-9}$. La tomografía axial computada se utiliza en aquellos casos más complejos donde se necesita mayor precisión o en casos de neoplasias en que se requiere etapificación. Se ha descrito que detecta hasta un $71 \%$ de los factores anatómicos responsables de una invaginación ${ }^{5}$. Otra alter- 
nativa diagnóstica es la colonoscopia, la cual nos permite ver una lesión polipoidea ubicada en el ciego donde debiese verse el apéndice cecal $^{10-12}$.

El tratamiento de la invaginación intestinal recurrente contempla la desinvaginación neumática, la hidrostática y el tratamiento quirúrgico con reducción manual o resección intestinal. La recurrencia luego de un tratamiento quirúrgico es del $1-3 \%{ }^{13-16}$ y alcanza un 10$15 \%$ luego de la reducción neumática o hidrostática $^{17-19}$. Existen estudios que sugieren que la desinvaginación neumática es más ventajosa que la hidrostática debido a que permite una reducción más fácil, menos radiación, menos morbilidad en caso de perforación intestinal y menor incidencia de recurrencias ${ }^{18-20}$. Como se pudo observar en nuestro caso clínico, tanto la desinvaginación neumática como la hidrostática de una invaginación secundaria a un remanente apendicular es posible, sin embargo, se presentaron recurrencias lo que significó mayor morbilidad para el paciente y costos emocionales y económicos para su familia. Considerando estos antecedentes creemos que en casos de invaginación intestinal recurrente el tratamiento de elección es el quirúrgico, ya sea con técnica abierta o laparoscópica ${ }^{21}$.

\section{Referencias}

1.- Osses J, Mariño L, Gabrielli F, et al: Invaginación recurrente múltiple por pólipo juvenil. Arch Argent Pediatr 2003; 101 (4): 265-9.

2.- Arora A, Caniano D, Hammond S, Besner G: Inversion appendectomy acting as a lead point for intussusception. Pediatr Surg Int 2008; 24: 1261-4.

3.- Dunn D, Beilman G: Surgical infections. En: Brunicardi $\mathrm{CF}$ et al. (eds) Schwartz principles of surgery. (2005) McGraw-Hill, New York, pp 109-27.

4.- Edebohls GM: Inversion of the vermiform appendix. Am J Med Sci (1895) 109: 650-6.

5.- Navarro O, Dugougeat F, Kornecky A, Shuckett B, Alton $D$, Daneman A: The impact of imaging in the management of intussusception owing to pathologic leadpoints in children. Pediatr Surg Int 2008; 24: 1261-4.
6.- Blakelock R, Beasly S: The clinical implications of nonidiopathic intussusception. Pediatr Surg Int 1997; 14: 163-7.

7.- Daneman A, Alton D, Lobo E, Gravett J, Kim P, Ein S: Patterns of recurrence in intussusception in children: A 17-year review. Pediatr Radiol 1998; 28: 913-9.

8.- Daneman A, Alton DJ, Shuckett B, et al: Intussusception: lead points and recurrences \pm role of the air enema and sonography. Poster exhibit at Third International Pediatric Radiology Meeting (1996). Boston, Massachusetts, USA.

9. Daneman A, Myers $M$, Shuckett B, et al: Sonographic appearances of inverted Meckel diverticulum with intussusception. Pediatr Radiol 1997 27: 295-8.

10.- Tavakkoli H, Sadrkabir SM, Mahzouni P: Colonoscopic diagnosis of appendiceal intussusception in a patient with intermittent abdominal pain: a case report. World J Gastroenterol 2007; 13: 4274-7.

11.- Ryu BY, Kim TH, Jeon JY, et al: Colonoscopic diagnosis of appendiceal intussusception: a case report. J Korean Med Sci 2005; 20: 680-2.

12.- Duncan JE, DeNobile JW, Sweeney WB: Colonoscopic diagnosis of appendiceal intussusception: case report and review of the literature. JSLS 2005; 9: 488-90.

13.- Soper RT, Brown MJ: Recurrent acute intussusception in children. Arch Surg 1964; 89: 188-98.

14.- Eklof $O$, Reiter $S$ : Recurrent intussusception analysis of a series treated with hydrostatic reduction. Acta Radiol Diag 1978; 19: 250-8.

15.- Kenigsberg K, Lee J, Stein H: Recurrent acute intussusception. Pediatrics 1979; 53: 269-70.

16.- Ein SH. Recurrent intussusception in children. J Pediatr Surg 1975; 10: 751-5.

17.- Champoux AN, Del Beccaro MA, Nazar-Stewart V, et al: Recurrent intussusception risks and features. Arch Pediatr Adolesc Med 1994; 148: 474-8.

18.- Stringer DA, Ein SH: Pneumatic reduction: advantages, risks and indications. Pediatr Radiol 1990; 20: 475-7.

19.- Renwick AA, Beasley SW, Phelan E: Intussusception: recurrence followings (oxygen) enema reduction. Pediatr Surg Int 1992; 7: 361-3.

20.- Beasley SW, Auldist AW, Stokes KB: Recurrent intussusception: barium or surgery? Aust N Z J Surg 1987; 57 : 11-4.

21.- Van der Laan M, Bax NM, Van der Zee DC, et al: The role of laparoscopy in the management of childhood intussusception. Surg Endosc 2001; 15 (4): 373-6. 